An online $E E G \mathrm{BCl}$ based on covert visuospatial attention in absence of exogenous stimulation

This article has been downloaded from IOPscience. Please scroll down to see the full text article.

2013 J. Neural Eng. 10056007

(http://iopscience.iop.org/1741-2552/10/5/056007)

View the table of contents for this issue, or go to the journal homepage for more

Download details:

IP Address: 128.178.22.133

The article was downloaded on 06/08/2013 at 17:13

Please note that terms and conditions apply. 


\title{
An online EEG BCI based on covert visuospatial attention in absence of exogenous stimulation
}

\author{
L Tonin, R Leeb, A Sobolewski and J del R Millán \\ Chair in Non-Invasive Brain-Machine Interface, École Polytecnique Fédérale de Lausanne, Lausanne, \\ Switzerland \\ E-mail: robert.leeb@epfl.ch
}

Received 16 April 2013

Accepted for publication 17 July 2013

Published 5 August 2013

Online at stacks.iop.org/JNE/10/056007

\begin{abstract}
Objective. In this work we present - for the first time - the online operation of an electroencephalogram (EEG) brain-computer interface (BCI) system based on covert visuospatial attention (CVSA), without relying on any evoked responses. Electrophysiological correlates of pure top-down CVSA have only recently been proposed as a control signal for BCI. Such systems are expected to share the ease of use of stimulus-driven BCIs (e.g. P300, steady state visually evoked potential) with the autonomy afforded by decoding voluntary modulations of ongoing activity (e.g. motor imagery). Approach. Eight healthy subjects participated in the study. EEG signals were acquired with an active 64-channel system. The classification method was based on a time-dependent approach tuned to capture the most discriminant spectral features of the temporal evolution of attentional processes. The system was used by all subjects over two days without retraining, to verify its robustness and reliability. Main results. We report a mean online accuracy across the group of $70.6 \pm 1.5 \%$, and $88.8 \pm 5.8 \%$ for the best subject. Half of the participants produced stable features over the entire duration of the study. Additionally, we explain drops in performance in subjects showing stable features in terms of known electrophysiological correlates of fatigue, suggesting the prospect of online monitoring of mental states in BCI systems. Significance. This work represents the first demonstration of the feasibility of an online EEG BCI based on CVSA. The results achieved suggest the CVSA BCI as a promising alternative to standard BCI modalities.
\end{abstract}

(Some figures may appear in colour only in the online journal)

\section{Introduction}

Current electroencephalogram (EEG) based brain-computer interface (BCI) systems can be divided into two categories: those which are stimulus-driven and those relying on voluntary modulations of ongoing neuronal activity. The former are mainly based on electrophysiological responses to exogenous stimulation (e.g. the P300 component of the event-related potential [1] or steady state visually evoked potential [2]) and the latter on self-initiated, or purely top-down, modulations of motor imagery (MI) related brain rhythms [3, 4]. On one hand, stimulus-driven BCI systems are generally robust and require a short training phase. However, continued passive stimulation has a tendency to become uncomfortable for most subjects, especially over longer periods of time. Furthermore, recent studies demonstrate that such BCIs depend considerably on gaze direction, which represents a limitation for locked-in patients [5]. On the other hand, voluntary modulation of brain activity — as in the case of MI-allows a more direct and active BCI experience, but the training phase may last longer [6]. Over the last few years both types of system have been widely exploited to demonstrate the possibility of using EEG based BCIs for restoring the control and communication capabilities of disabled people [7-9].

Recently, several groups started investigating the feasibility of BCI applications based on covert visuospatial 

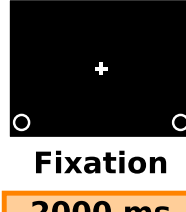

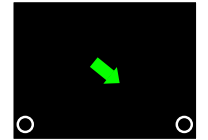

Cue

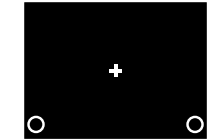

Covert attention

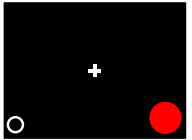

Feedback

\section{0 ms $\quad$ Classification $\quad 3000$ ms}

Figure 1. Schematic trial representation. A fixation cross appears $2000 \mathrm{~ms}$ before the cue. The cue (100 ms duration) indicates the locus of attention. After 3000-5000 ms the red circle appears at one of the target locations for $1000 \mathrm{~ms}$ indicating the classification output (feedback). Classification is based only on the data from the first $3000 \mathrm{~ms}$ after cue onset.

attention (CVSA). CVSA refers to the process of focusing attention on different regions of the visual field without overt eye movements [10]. Flexibility and intuitiveness make CVSA a promising candidate for BCI applications, especially in the field of navigation devices (i.e. wheelchairs, telepresence robots). Several electrophysiological correlates of CVSA have been discovered by basic neurophysiological research. On one hand, they comprise time-locked phenomena related to stimulus processing. Particularly, early components of visual evoked potentials $(\mathrm{P} 1, \mathrm{~N} 1)$ have been shown to be enhanced if evoked by a stimulus appearing at the attended location $[11,12]$. These enhancements are construed to reflect an attentional amplification of localized sensory information at the level of visual pathways [11]. Such a modality has been already explored for control of BCI communication devices (e.g., P300-Speller [13], Hex-O-Spell [14]).

However, electrophysiological correlates of CVSA also include asynchronous, purely top-down processes. Selective modulations of the parieto-occipital $\alpha$-activity in scalp EEG have been shown to reflect endogenous shifts in the locus of attention [15-18]. They are thought to rely primarily on distributed $\alpha$-synchronizations (up-regulations), serving as active suppressors of unattended locations [17]. Initial offline studies aimed at possible BCI usage followed these basic findings. They have demonstrated the potential feasibility of single-trial (real-time) classification - the hallmark of BCI operation [19, 20]. However, an operational online BCI based on self-initiated, top-down EEG correlates of CVSA has not been built until now.

It should be noted here that research in this regard has been done using other brain imaging techniques which allow greater spatial resolution (i.e., with magnetoencephalogram [21, 22]). Furthermore, a few studies have demonstrated the possibility of using physiological correlates of CVSA as a BCI-control signal with functional magnetic resonance imaging [23, 24]. In particular, in [24] the authors demonstrated the possibility of decoding CVSA online from the blood oxygen level dependent contrast and using it to control a telepresence robot.

In our preliminary research [20] we have investigated the best classification method for decoding shifts of locus of CVSA from EEG in the case of pure top-down modulation. We devised a time-dependent (evolving) approach to feature selection and classification; offline results have shown it to be superior to standard methods reported previously in the literature. The main purpose of this study is to present the first online EEG BCI based on CVSA, which operates without relying on any stimulus-driven responses.

\section{Material and methods}

\subsection{Participants}

Eight healthy volunteers (from 24 to 38 years old, median 27.5 years) with normal or corrected-to-normal vision participated in this study (S1-8). Subjects $\mathrm{S} 1-3$ were already involved in previous research [20], while S4-8 did not have any previous experience with CVSA paradigms. The study was approved by the local ethics committee and carried out in accordance with the principles of the Declaration of Helsinki.

\subsection{Visuospatial attention protocol}

In this study we exploited a modified version of Posner's spatial cueing task [10] (see figure 1). The same visual paradigm has been exploited in our previous CVSA offline study [20]. A white fixation cross in the center of a computer screen (spanning $3.12^{\circ}$ of the visual field) and two white circles of $3.12^{\circ}$ positioned at the bottom-left and bottom-right locations, $12^{\circ}$ off center, were displayed continuously. After $2000 \mathrm{~ms}$ of fixation, a cue (arrow) was displayed for $100 \mathrm{~ms}$ indicating on which of the two target locations (circles) the subject is supposed to focus their attention. Subjects were instructed to perform the task without overt eye movements. After 3000$5000 \mathrm{~ms}$ of sustained covert attention, a red disc appeared over one of the target locations, indicating the result of the classification (feedback) and signifying the end of the trial. (During the calibration phase the feedback always appeared at the correct, i.e. cued, location.) The random duration of the covert attention period was exploited to avoid the subject adaptating to the protocol pace. During the covert attention period no external stimuli were provided to the subjects. Subjects were instructed to blink or move their eyes only after the end of the trial.

\subsection{Experimental design}

Each subject participated in two recording sessions separated by 1-2 days. On the first day subjects performed four calibration runs (totaling 160 trials, 40 per run). The calibration phase lasted on average $26.5 \pm 8.5 \mathrm{~min}$. After a break $(33.7 \pm 7.6 \mathrm{~min})$, subjects performed four online runs (totaling 


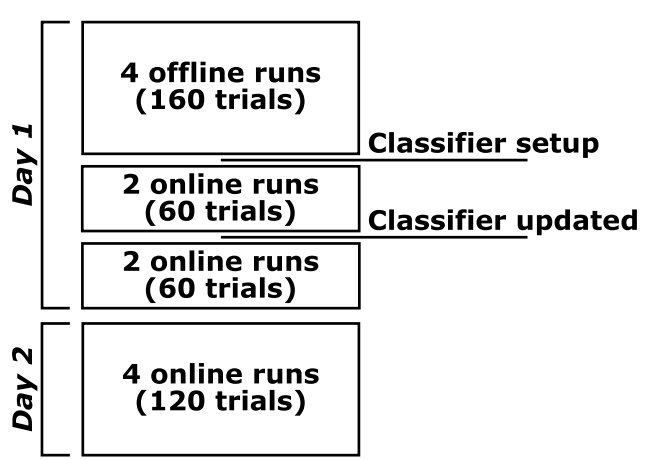

Figure 2. The calibration and online phases over the two recording days. On the first day subjects performed four offline and four online runs. After the first two online runs the classifier was updated with the new data. Following this update, the classifier remained unchanged for the rest of the recordings.

120 trials, 30 per run). The classifier used in the online runs was built with the data from the calibration phase and then updated with the first two online runs. On the second day the subjects performed four additional online runs (totaling 120 trials, 30 per run) using the same classifier, i.e. from the previous day (figure 2). In total, each subject performed 400 trials across the two recording days. Trials were randomly shuffled between the two classes.

\subsection{Data acquisition and preprocessing}

EEG signals were acquired with an active 64-channel system (Biosemi, Amsterdam, the Netherlands) at $2048 \mathrm{~Hz}$ sampling frequency (bandwith DC-400 Hz). The 64 electrodes were placed according to the standard international 10-20 system. After acquisition, the EEG data of a given trial was lowpass filtered (cut-off frequency at $200 \mathrm{~Hz}$ ) and downsampled to $512 \mathrm{~Hz}$. Thereafter, the envelope of the signals (absolute value of Hilbert transformation) was computed for seven frequency sub-bands in the $\alpha$ range (Butterworth filters of order $4,3 \mathrm{~Hz}$ windows, centered at integer values from 8 to $14 \mathrm{~Hz}$ ). Subsequently, a Laplacian spatial filter was applied. We used a configuration based on the closest neighbor electrodes (distance $<5 \mathrm{~cm}$ ) weighting the contribution of each neighbor according to the distance from the target electrode (inverse proportion) - an extension of the standard Laplacian configuration proposed in the literature [25]. Finally, we cropped the channels in the parieto-occipital regions of the brain (17 electrodes: P7, P5, P3, P1, Pz, P2, P4, P6, P8, PO7, $\mathrm{PO} 3, \mathrm{POz}, \mathrm{PO} 4, \mathrm{PO} 8, \mathrm{O} 1, \mathrm{Oz}, \mathrm{O} 2)$.

Concurrently with the EEG we recorded gaze direction with a remote eyetracking device (SensoMotoric Instrument $\mathrm{GmbH}$, Teltow, Germany). The two recording systems were synchronized by means of a direct TCP connection. Since our eye-tracking system worked at $60 \mathrm{~Hz}$, the data was resampled by means of linear interpolation to match the EEG sampling rate $(512 \mathrm{~Hz})$.

\subsection{Online BCI classification}

In order to discriminate between the two classes (the left or right locus of attention) we adopted a time-dependent classification method. The advantages of this approach with respect to standard methods have already been reported in [20]. Briefly, the classification of every trial was based only on the data from the first $3000 \mathrm{~ms}$ after cue onset. (Longer trials served only to reduce possible expectancy effects.) This period was split into consecutive non-overlapping windows of $150 \mathrm{~ms}$. For each window we built a quadratic discriminant analysis classifier using as input the most discriminative features (frequency-channel pairs) based on their Fisher score values computed across the two classes on the calibration data (for details see [20]). During online runs each classifier analyzed its corresponding window sequentially: we accumulated the posterior probabilities of each time window in a Bayesian framework in order to make the final decision about the trial [26]. The online accuracy of the BCI was evaluated in terms of the percentage of correctly classified trials.

\subsection{Offline analysis}

After the completion of the online sessions, the data was additionally analyzed offline in order to further investigate the performance of the system. In particular, we evaluated (i) the possible influence of eye movements, (ii) the stability of the features across the different runs/days and (iii) possible electrophysiological correlates of cognitive processes or physiological states that may explain the variation of the BCI accuracy.

Gaze control. Precise control of gaze direction becomes fundamental when studying CVSA and BCI based on it. Using eye-tracker data we: (i) quantified the incidences of overt gaze visits to the target locations and (ii) investigated the possible influences of eye movements on the classification.

We defined two circular regions of interest (RoI) covering $14.6^{\circ}$ of the visual field, centered on the target locations. We conservatively considered that a target location received an overt glance (saccades) if the subject's gaze visited the corresponding RoI continuously for at least $50 \mathrm{~ms}$. The percentage of trials with overt gaze visits to target locations proved to be very low; detailed results are provided in section 3.2.

However, to additionally verify whether any shifts in gaze direction could influence, or account for, our BCI classification results, we performed classification of the trials based solely on eye movements. From eye-tracker data we extracted the horizontal component of gaze location. Setting the fixation cross as the origin (zero), the integral of the horizontal coordinate values over a trial gave us a metric specifying which side of the screen the subject's gaze was principally attracted to. The trials were classified as left or right based on this metric. Subsequently, for each subject a $\phi$ coefficient was calculated between a vector of gaze-based trial-by-trial classifications (correct or incorrect, 1 or 0 ) and a similar vector for BCI classifications. The $\phi$ coefficient is a measure of association between two variables; it can be 
(a) Online classification performance

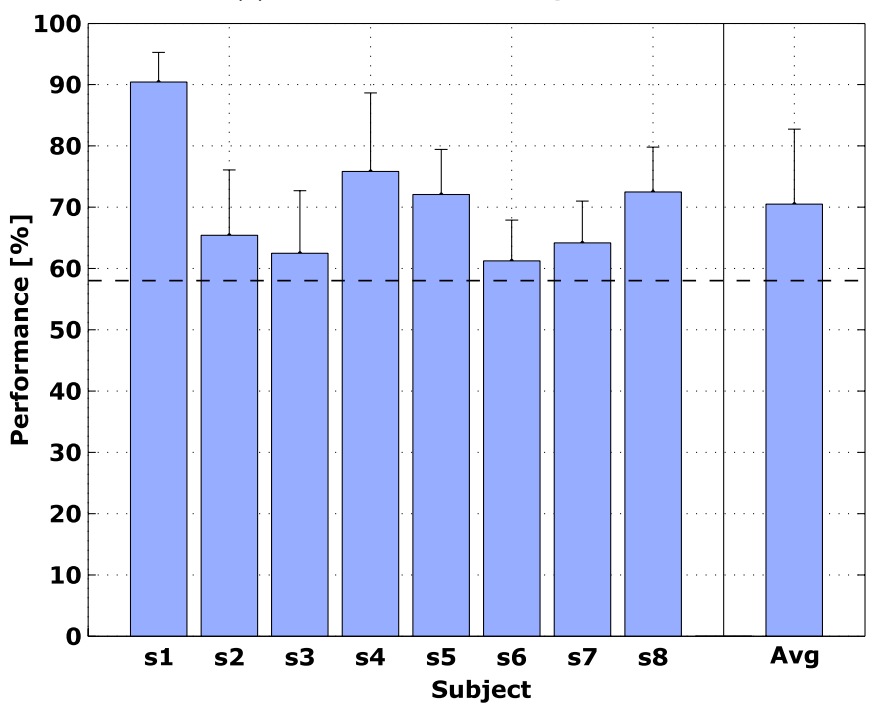

(b) Temporal evolution of online classification performance

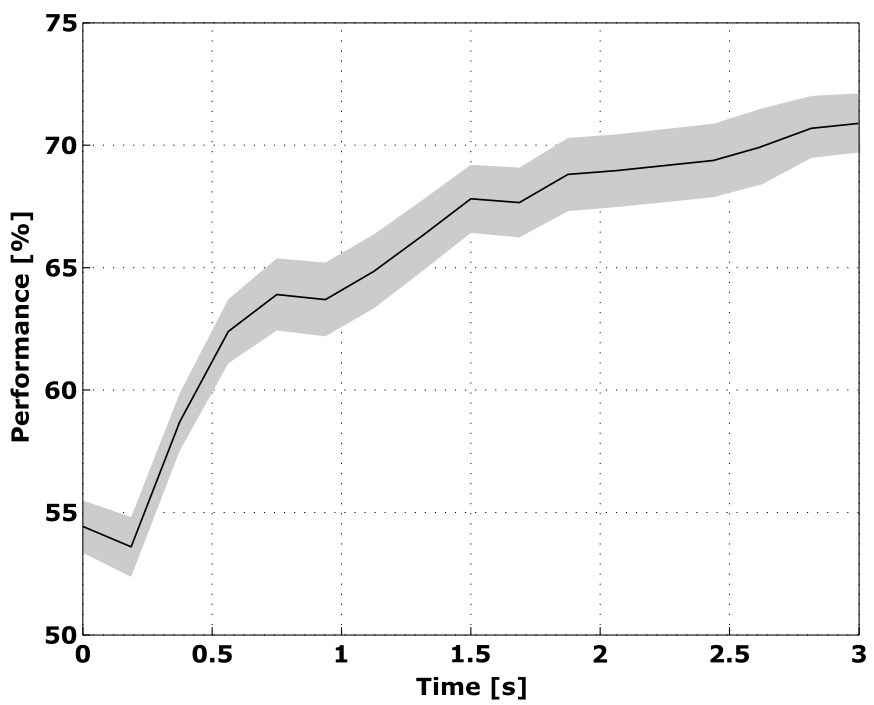

Figure 3. (a) Online performance of each subject averaged over the two days of the experiment. Error bars denote standard deviation of the mean. The broken line indicates the chance level of $p<0.05$. (b) Evolution of the accuracy over the attention period (average over subjects and days). The shaded area denotes the SEM.

considered an equivalent of Pearson's correlation coefficient for binary variables. High association between gaze-based and BCI classifications would mean that both perform well, or fail, in the same trials, thus suggesting that both rely on the same physiological processes-correlates of overt shifts in gaze direction, or possibly even obscure electroocular artifacts. Low or no association would demonstrate that BCI classification detects cortical processes which are overall independent of incidental gaze shifts.

Variations in performance. A fundamental requirement for a BCI system is stability of performance over different runs or days. For this reason we did not limit our study to a single session, nor did we retrain the classifier for the second recording day. However, a posteriori offline investigations into (practically inevitable) performance variability can provide insights into its causes. A drop in online BCI accuracy may be caused by two factors: (i) the subject stopped producing discriminative electrophysiological patterns in response to task requirements, or (ii) the subject still generates discriminative electrophysiological patterns, but different to the ones used in construction of the classifier (feature shift). We believe that any meaningful explanation of variability in BCI performance must be preceded by a dissociation of these confounding factors. In the first case explanations may be sought in the physiological state of the subject (e.g. fatigue, excessive workload); in the case of feature shift the drop in performance is primarily a learning, or machine learning challenge.

For the purpose of making this dissociation, we introduce a straightforward metric, which we term the modulation index, assessing how much a subject modulated his/her overall EEG features during online BCI operation-irrespective of the classifier used during a particular run. The modulation index is defined as follows. Firstly, we compute the Fisher score values across the two classes for each feature (frequency bin/EEG channel pair) and each online run completed by the subject.
Then, we sum the Fisher score values of all features (here: 17 channels, 7 frequency bands) for each run, obtaining one value per run.

For each subject we correlated the vector of modulation indices with the performances (percentages of correct trials) achieved in each run. Performances are directly related to the values of the features selected to train the classifier, while the modulation index is representative of the whole feature space. Thus, performances and modulation indices showing similar dynamics across runs suggest no shifting of the features. Conversely, a decrease in performance accompanied by a stable, or increasing, modulation indices indicates that new, or shifted, discriminant features (different from those used as input for the classifier) are produced by the subject in consecutive online runs. Correlation coefficients between the modulation indices and BCI performances may accordingly present two different cases: (i) high positive correlationperformances are following the modulation index across runs, features are stable; (ii) low/negative correlation-features are not stable and are shifting between runs.

Due to relatively small sample sizes, the statistical significance of all correlation coefficients calculated across runs has been conservatively verified with a non-parametric, empirical test. In each case we shuffled the relevant values randomly 1000 times, obtaining a representation of the random distribution of correlation coefficients bootstrapped from real data. Subsequently, we inferred statistical significance levels ( $p<0.05$ and $p<0.01$ ) from the 95th and 99th percentiles of the distribution.

\section{Results}

\subsection{Online BCI performances}

Figure 3(a) shows the online performances for each subject averaged over the two days of the experiment. All subjects 
(a) Trials involving gaze shifts

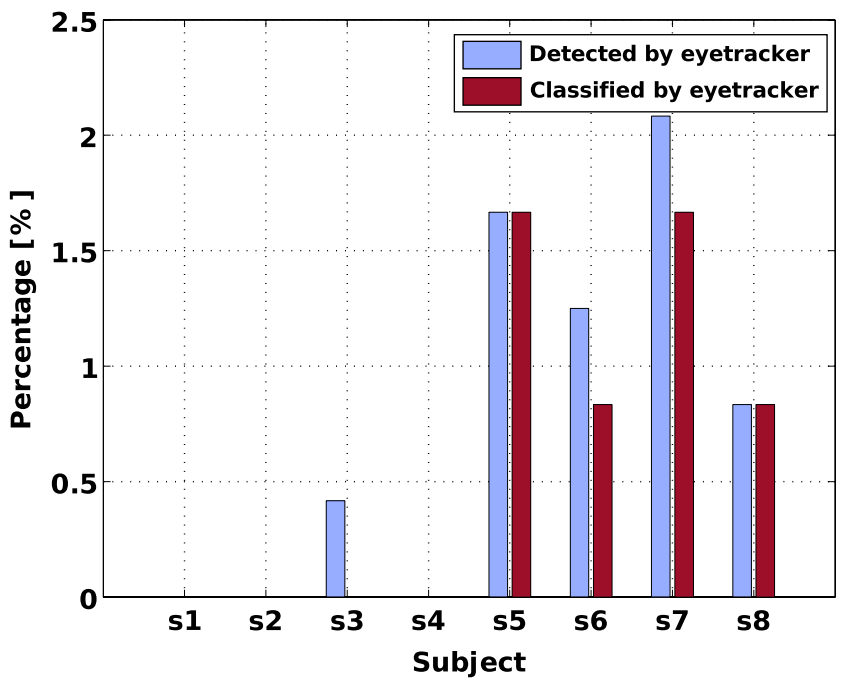

(b) Correlation between BCl and gaze-based classification

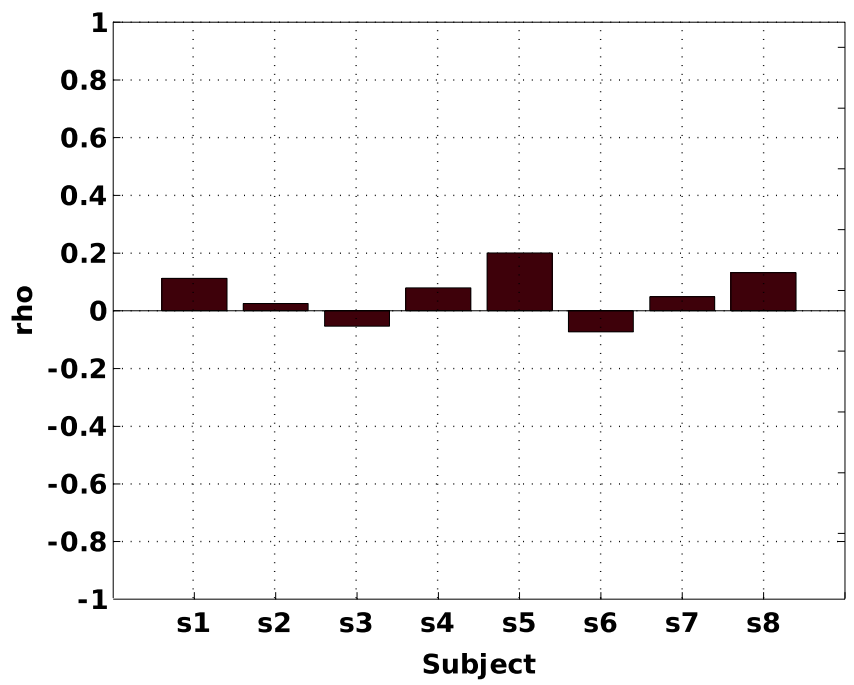

Figure 4. Overt eye movements. (a) Percentage of trials involving overt gaze shifts: (i) towards either one of the target locations (blue bars); (ii) towards just the cue target location (red bars). (b) Correlations ( $\phi$ coefficients) between the trial-by-trial accuracy of classification based on the horizontal component of eye saccades and BCI; none significant at $p<0.05$.

performed better than a chance level of $p<0.05$ [27]. The overall accuracy across subjects and runs was $70.6 \pm 1.5 \%$ (mean \pm standard error of the mean (SEM)). In addition, four subjects (s1, s4, s5 and s8) show an average accuracy above 70\% - the level required for practical BCI use [28]. Notably, among them only subject $\mathrm{s} 1$ had previous experience with CVSA. Furthermore, such a level of performance was achieved with one short calibration phase of $\sim 30 \mathrm{~min}$ and did not require any extended training of the subjects.

Figure 3(b) depicts the evolution of accuracy computed a posteriori offline sample by sample over the trial period. Two aspects are noteworthy: firstly, the performance based just on the first $\sim 500 \mathrm{~ms}$ would not exceed chance level. This fact verifies that classification is not driven by the event-related response to the cue (the event-related response). Secondly, the curve essentially increases monotonically, demonstrating proper operation of the evidence accumulation framework.

In table 1 we report the results for each run. Generally, subjects s1, s3, s6 and s7 have stable performance across both days (standard deviation below 10\%). Among them subject s1 achieved the highest performance $(88.8 \pm 5.8 \%$ s.d.), while subjects s3, s6 and s7 displayed lower performances during both days $(63.6 \pm 8.4 \%, 65 \pm 5.3 \%$ and $64.6 \pm 7.9 \%$, respectively). Interestingly, for subjects s5 and s8, we can identify a strong improvement across runs with a final accuracy of $75.8 \%$ and $79.2 \%$ during the second day (i.e., increments of $5.8 \%$ and $9.2 \%$, respectively). Conversely, subjects s2 and s4 have good performances on the first day (accuracy of $75.8 \%$ and $83.3 \%$ ) whereas they suffer a large drop during the second recording session (decrements of $23.3 \%$ and $25.5 \%$, respectively). We investigate the possible factors responsible for this drop in sections 3.3 and 3.4.

\subsection{Gaze shifts}

In figure 4(a) we present the percentage of trials that involved overt gaze direction shifts towards either one of the target locations (blue bars). This percentage is very low for all subjects: $0.78 \%$ on average, maximum $<3 \%$; it is $0 \%$ for subjects s1, s2 and s4 and below $1 \%$ for s3 and s8. As expected, most of the eye movements were directed towards the cued target location (red bars in the figure). Given the small number of contaminated trials it can be assumed that gaze shifts had a negligible impact in the classification accuracy. However, additionally we have investigated the association between classification based on prevailing gaze direction in a given trial and EEG-based BCI classification (see section 2.6 for methodological details). We found low or no correlation for all subjects (figure 4(b)), further evidencing that BCI classification detects cortical processes which are independent of any occurring gaze shifts.

\subsection{Stability of features}

Data from the online runs was additionally analyzed a posteriori in order to investigate the observed variations in performance in subjects across different runs. As already proposed in section 2.6, a drop in online BCI performance may be caused by: (i) the subject not producing discriminative electrophysiological patterns, or (ii) feature shift (i.e., a change in discriminative patterns). Dissociation of these two confounding factors is essential, as in the first case the best course of action may be to seek reasons in the physiological state of the subject (e.g., cognitive fatigue), while feature shifts without a drop in overall discriminability should probably be approached as signal non-stationarities, and remedied with machine learning techniques, or subject training.

To address this distinction, we proposed a simple metric termed the modulation index, which quantifies the subjects' capability to generate task-relevant discriminative features irrespective of the particular classifier used during BCI operation. To assess the stability of features we correlated the modulation index with BCI performance across runs (please 
Table 1. The online performance (\%) for each subject across runs. The runs with a classification accuracy above $70 \%$ are in bold. The final two columns show the average performance of the two days.

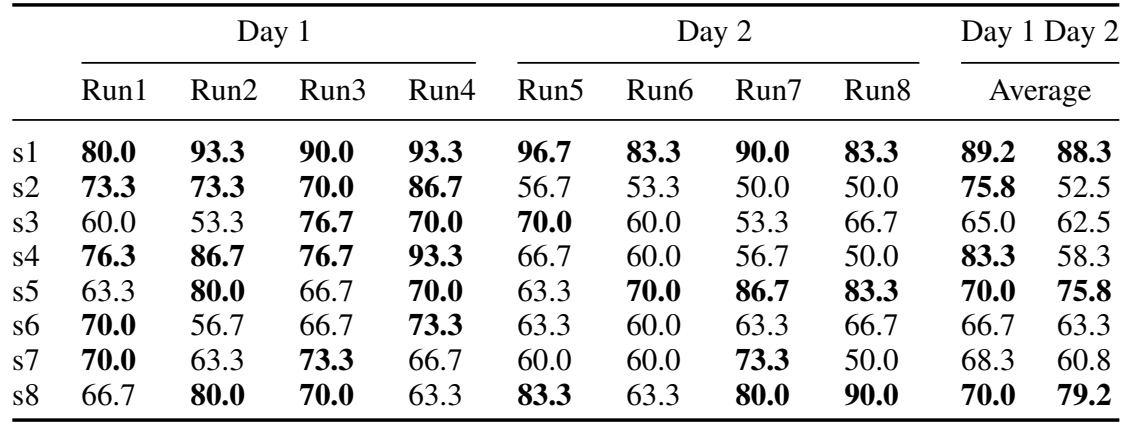

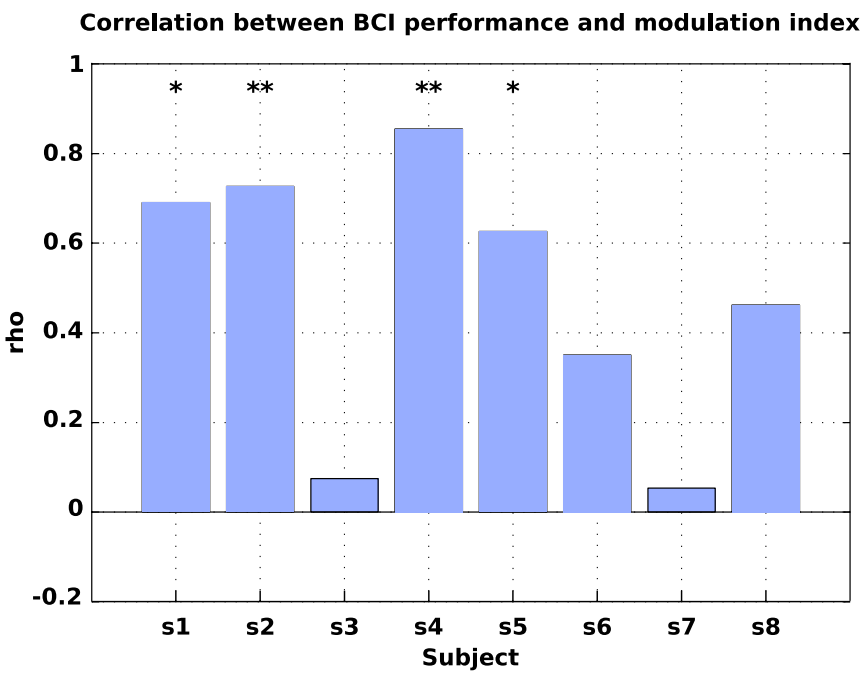

Figure 5. Correlation between BCI performance and modulation index. The statistical significance of the correlation coefficients is reported $(* p<0.05, * * p<0.01)$.

see section 2.6 for computational details and rationale). We report the results in figure 5 . The correlation shows a general positive trend and is statistically significant for four subjects (s1, s2, s4 and s5). Thus, for these subjects performance follows the evolution of the modulation index, suggesting that features are stable across runs and days. This does not apply to subjects s3, s6 and s7, where the correlation coefficients are low. We can conjecture that these subjects were not able to generate a stable set of features across different runs. Subject s8 shows considerable positive correlation, but the significance of the correlation did not survive our conservative statistical test. Nevertheless, this subject shows high (and increasing) performance across the recording sessions and this is possible only if the features selected during the calibration were still discriminable in the online runs. For this reason, we assume a generation of new extra features rather than an instability of the original ones.

\subsection{EEG correlates of performance degradation}

Above we identified four subjects (s1, s2, s4 and s5) with stable features across different runs. Two of them (s2 and s4) showed a consistent drop in performance during the second day (see table 1), which thus could not be attributed to feature shift.
Consequently, we searched for correlates of these decrements among electrophysiological effects reported to accompany plausible cognitive or physiological states such as fatigue, excessive workload, drowsiness, etc. Studies in this respect seem to converge on a limited subset of phenomena: primarily changes in EEG $\delta, \theta$ and/or $\alpha$ activity over frontal cortical areas [29-31].

We have calculated the correlation coefficients between the frontal power in these bands $(1-4,4-8$ and 8-12 Hz, respectively, averaged of electrodes: Fp1-2, Af7-8, F7-8, FT7-8) and the modulation index across runs for each of the above four subjects. Remarkably, it is only subjects s2 and s4 who exhibit significant correlations of the modulation index with frontal EEG power in $\delta(\phi=-0.74, p<0.01)$, $\theta(\phi=-0.77, p<0.01), \alpha(\phi=-0.69, p<0.01)$ and in $\delta(\phi=-0.55, p<0.05)$ bands, respectively. Their ability to modulate BCI-relevant EEG features, and thus their BCI performances, is decreasing (see figure 5 and table 1) as the frontal EEG spectral power in the relevant bands increases. This finding, in accordance with neuroscientific literature [30], directly supports the hypothesis that, in the case of s 2 and s4, mental fatigue-related processes are the cause of the drop in performance.

\section{Discussion}

In this study we demonstrated for the first time the online operation of an EEG BCI based on CVSA not relying on any external stimulation, but directly tapping into the brain's top-down attentional processes. Notably, we also report the performance and stability of such BCI over different days.

Online classification accuracy of $70.6 \pm 1.5 \%$ (averaged across subjects and recording sessions) proves CVSA to be a valid control signal for BCI applications [28]. This result did not require any extended training of the subjects, but only a 30 min calibration session. Such a level of online performance has been achieved through the implementation of the time-dependent classification approach proposed in [20]. In that previous work we demonstrated offline that more detailed analysis in both frequency and time domains can improve classification accuracy by better capturing the temporal dynamics of EEG correlates of the orienting of visual attention. The results achieved in this online study confirm the validity of our classification approach. 
We also verified the stability of the system over different days. Encouragingly, for most subjects the achieved performance was fairly consistent, however we did encounter considerable variations (see table 1). As an addition to the main goal of this paper, we attempted to shed some light on the underlying factors of this variability. We believe that to meaningfully explain variations in BCI performance it is first necessary to dissociate between two confounding factors: (i) changes in BCI performance caused by shifting, or unstable, features invalidating the classifier, and simply (ii) lower capability of the subject during a particular run due to current physiological state, for example mental fatigue. To this end, in an a posteriori analysis of the online data, we have compared the subjects' ability to generate task-relevant discriminative features irrespective of the particular classifier used with the achieved BCI performance. Considerable positive correlations between classifier-independent modulation indices and online accuracy obtained for most subjects suggest that these subjects generated stable features over different recording sessions, and thus most drops in performance have to be attributed to other factors. Two of these subjects (s2 and s4) reported a drop of performance during the second day. For both of these subjects we found that the performance variations were inversely correlated with the EEG's spectral power in the $\delta$ band over frontal scalp areas (as well as frontal $\theta$ and $\alpha$ power for subject s2). As increases in these frontal oscillations have been consistently reported as accompanying mental fatigue (see [30] for a recent comprehensive review) this clearly suggests a physiological reason of the performance drops in these subjects, rather than BCI design. This inference is far from being proven given the number of subjects involved in the study as well as the lack of other measures of physiological state. Nevertheless, we present it as an interesting approach to explaining variability in BCI performance, as well as an argument for possible online monitoring of users' physiological states.

The proof-of-concept contained in this paper opens up questions regarding possible future applications of EEG BCIs based on CVSA. Inherent gaze independence of CVSA makes it a promising alternative for completely locked-in patients, displaying no overt eye movements. Its intuitiveness-natural attraction toward regions or objects of interest in the visual field-makes it an interesting candidate for BCI-driven navigation devices (e.g., telepresence robots [9, 24]), as well as simple yes-no communication. Lack of tiresome artificial stimulation may prove it more suitable for use over longer periods of time, as it allows a more engaging and direct operation. However, the fatiguing effects of prolonged attentional demands remain to be appraised.

Another interesting prospect lies in coupling CVSA with other BCI control signals in a hybrid framework-particularly with another major non-time-locked paradigm, i.e., MI. Advantages include the fact that both rely on independent EEG correlates, with isolated scalp distributions. The hybrid architecture could be developed in a complementary or supplementary schema in order to increase the number of commands or to decrease the uncertainty of the existing ones.

\section{Acknowledgments}

This work is supported by the European ICT Programme Project TOBI (FP7-224631). This paper only reflects the authors' views and funding agencies are not liable for any use that may be made of the information contained herein.

\section{References}

[1] Farwell L A and Donchin E 1988 Talking off the top of your head: toward a mental prosthesis utilizing event-related brain potentials Electroencephalogr. Clin. Neurophysiol. 70 510-23

[2] Middendorf M, McMillan G, Calhoun G and Jones K S 2000 Brain-computer interfaces based on the steady-state visual-evoked response IEEE Trans. Rehabil. Eng. 8 211-4

[3] Pfurtscheller G, Brunner C, Schlögl A and Lopes da Silva F H $2006 \mu$ rhythm (de)synchronization and EEG single-trial classification of different motor imagery tasks NeuroImage 31 153-9

[4] Millán J d R, Mouriño J, Franzé M, Cincotti F, Varsta M, Heikkonen J and Babiloni F 2002 A local neural classifier for the recognition of EEG patterns associated to mental tasks IEEE Trans. Neural Netw. 13 678-86

[5] Brunner P, Joshi S, Briskin S, Wolpaw J R, Bischof H and Schalk G 2010 Does the 'P300' speller depend on eye gaze? J. Neural Eng. 7056013

[6] Leeb R, Perdikis S, Tonin L, Biasiucci A, Tavella M, Molina A, Al-Khodairy A, Carlson T and Millán J d R 2013 Transferring brain-computer interface skills: from simple BCI training to successful application control J. Artif. Intell. Med. at press

[7] Piccione F, Giorgi F, Tonin P, Priftis K, Giove S, Silvoni S, Palmas G and Beverina F 2006 P300-based brain computer interface: reliability and performance in healthy and paralysed participants Clin. Neurophysiol. 117 531-7

[8] Nijboer F et al 2008 A P300-based brain-computer interface for people with amyotrophic lateral sclerosis Clin. Neurophysiol. 119 1909-16

[9] Tonin L, Carlson T, Leeb R and Millán J d R 2011 Brain-controlled telepresence robot by motor-disabled people EMBS'11: Proc. 33rd Annu. Int. Conf. of IEEE Engineering in Medicine and Biology Society (Boston, MA, USA) pp 4227-30

[10] Posner M 1980 Orienting of attention Q. J. Exp. Psychol. 32 3-25

[11] Hillyard S A and Anllo-Vento L 1998 Event-related brain potentials in the study of visual selective attention Proc. Natl Acad. Sci. 95 781-7

[12] Mishra J, Martínez A, Schroeder C E and Hillyard S A 2012 Spatial attention boosts short-latency neural responses in human visual cortex NeuroImage 59 1968-78

[13] Liu Y, Zhou Z and Hu D 2011 Gaze independent brain-computer speller with covert visual search tasks Clin. Neurophysiol. 122 1127-36

[14] Treder M S and Blankertz B 2010 (C)overt attention and visual speller design in an ERP-based brain-computer interface Behav. Brain Funct. 628

[15] Sauseng P, Klimesch W, Stadler W, Schabus M, Doppelmayr M, Hanslmayr S, Gruber W R and Birbaumer N 2005 A shift of visual spatial attention is selectively associated with human EEG alpha activity Eur. J. Neurosci. 22 2917-26

[16] Thut G, Nietzel A, Brandt S A and Pascual-Leone A 2006 Alpha-band electroencephalographic activity over occipital cortex indexes visuospatial attention bias and predicts visual target detection J. Neurosci. 26 9494-502 
[17] Rihs T A, Michel C M and Thut G 2007 Mechanisms of selective inhibition in visual spatial attention are indexed by $\alpha$-band EEG synchronization Eur. J. Neurosci. 25 603-10

[18] Treder M S, Schmidt N M and Blankertz B 2011 Gaze-independent brain-computer interfaces based on covert attention and feature attention J. Neural Eng. 8066003

[19] Treder M S, Bahramisharif A, Schmidt N M, van Gerven M and Blankertz B 2011 Brain-computer interfacing using modulations of alpha activity induced by covert shifts of attention J. Neuroeng. Rehabil. 824

[20] Tonin L, Leeb R and Millán J d R 2012 Time-dependent approach for single trial classification of covert visuospatial attention J. Neural Eng. 9045011

[21] van Gerven M, Bahramisharif A, Heskes T and Jensen O 2009 Selecting features for BCI control based on a covert spatial attention paradigm Neural Netw. 22 1271-7

[22] van Gerven M and Jensen O 2009 Attention modulations of posterior alpha as a control signal for two-dimensional brain-computer interfaces J. Neurosci. Methods 179 78-84

[23] Andersson P, Ramsey N F, Raemaekers M, Viergever M A and Pluim J P W 2012 Real-time decoding of the direction of covert visuospatial attention J. Neural Eng. 9045004

[24] Andersson P, Pluim J P W, Viergever M A and Ramsey N F 2012 Navigation of a telepresence robot via covert visuospatial attention and real-time fMRI Brain Topogr 26 177-85
[25] McFarland D J, McCane L M, David S V and Wolpaw J R 1997 Spatial filter selection for EEG-based communication Electroencephalogr. Clin. Neurophysiol. 103 386-94

[26] Beck J M, Ma W J, Kiani R, Hanks T, Churchland A K, Roitman J, Shadlen M N, Latham P E and Pouget A 2008 Probabilistic population codes for Bayesian decision making Neuron $601142-52$

[27] Müller-Putz G R, Scherer R, Brunner C, Leeb R and Pfurtscheller G 2008 Better than random? A closer look on BCI results Int. J. Bioelectromagn. 10 52-55

[28] Kübler A, Nijboer F, Mellinger J, Vaughan T M, Pawelzik H, Schalk G, McFarland D J, Birbaumer N and Wolpaw J R 2005 Patients with ALS can use sensorimotor rhythms to operate a brain-computer interface Neurology 64 1775-7

[29] Lal S K L and Craig A 2001 A critical review of the psychophysiology of driver fatigue Biol. Psychol. 55 173-94

[30] Borghini G, Astolfi L, Vecchiato G, Mattia D and Babiloni F 2013 Measuring neurophysiological signals in aircraft pilots and car drivers for the assessment of mental workload, fatigue and drowsiness Neurosci. Biobehav. Rev. at press

[31] Brouwer A, Hogervorst M A, van Erp J B F, Heffelaar T, Zimmerman P H and Oostenveld R 2012 Estimating workload using EEG spectral power and ERPs in the n-back task J. Neural Eng. 9045008 